Post- print de E. Aznar, A. Cros y L. Quintana. «Lectura y coherencia textual (Análisis de un texto narrativo)». Comunicación, lenguaje y educación. 17. 1993. pàg. 17-28.

DOI: $10.1080 / 02147033.1993 .10821058$

\title{
Lectura y Coherencia Textual (análisis de un texto narrativo):
}

La actividad que denominamos comúnmente "comprensión lectora" suele realizarse siguiendo un esquema que, con algunas variaciones, puede resumirse así: lectura en voz alta, uso del diccionario para las dificultades léxicas, preguntas para controlar si el significado elaborado por el alumno es el adecuado. A veces, la comprensión lectora comprende otras actividades como la redacción (resúmenes, reelaboraciones con finales alterados...), la gramática (análisis morfológico y sintáctico de frases del texto), incluso la historia de la literatura o de las ideas. Todos estos contenidos pertenecen a ámbitos científicos muy diversos y algunos de ellos sólo atañen parcialmente a la lectura. Entre otras razones, esta diversidad de contenidos y de objetivos obligan a evaluar la comprensión lectora de forma muy intuitiva.

Distintas aportaciones teóricas aparecidas recientemente cuestionan este esquema. Por una parte, la concepción de la lectura como proceso interactivo insiste en la autonomía de la lectura frente a otras habilidades, como el habla o la escritura. Esto ha puesto en duda la eficacia de procedimientos añejos como la lectura en voz alta. También se ha criticado, por ejemplo, relegar la solución de los conflictos interpretativos al uso del diccionario, puesto que, según esta misma concepción, es fundamental en el proceso lector saber elaborar hipótesis ante cualquier dificultad interpretativa, tenga carácter léxico o no. Implícitamente, la teoría interactiva ha puesto en duda la posibilidad de una comprensión única por más de un lector. En la segunda mitad del siglo, muchos teóricos de la crítica literaria han corroborado este rechazo a la posibilidad de una interpretación canónica y han insistido en la necesidad de aceptar distintas "lecturas" de un mismo texto.

Por otra parte, la lingüística del texto nos ha obligado a dirigir nuestra atención a un ámbito superior al de la oración y a considerar la comprensión de un texto como algo más amplio que la comprensión de las oraciones que lo componen. Un texto se caracteriza por su coherencia; la captación de esta coherencia es una condición indispensable para una buena lectura. 


\section{Propósito del presente artículo}

Es inevitable que todas estas aportaciones acaben asomando la cabeza por la escuela y que conviertan en obsoleto el viejo esquema de la "comprensión lectora" y del "análisis de textos". Una prioridad elemental es que su incorporación a la enseñanza no se realice de la forma desordenada con que se realizó la incorporación de la gramática generativa a la enseñanza de la gramática. En este sentido, el enseñante debe entender que no se trata de enseñar lingüística del texto o lectura interactiva, sino de aplicarlas a la recepción y producción de textos. Es fundamental también que el enseñante tenga claro que el esfuerzo que está a punto de realizar no es una necesidad impuesta por una moda sino un requisito indispensable para solucionar algunos problemas que el esquema tradicional no resolvía, lo cual es, al fin y al cabo, el móvil que hace progresar el conocimiento científico y, en un ámbito menos ambicioso, el motor de la formación permanente.

Nuestro propósito es mostrar aquí cómo una de las aportaciones citadas, la lingüística del texto, permite al enseñante calibrar el grado de coherencia de los textos que propone a los alumnos o de los que éstos le proponen a él. Expuesto de forma concreta, el enseñante puede encarar los siguientes objetivos:

a) ayudar a captar la coherencia del texto;

b) orientar actividades desarrolladas alrededor de la lectura, como son

1) localización y caracterización de los mecanismos de coherencia en un mismo texto;

2) formulación de preguntas control y de ejercicios que puedan ayudar al alumno a elaborar sus propias estrategias de lectura.

c) corregir e intervenir con un criterio sólido durante el proceso de escritura de los alumnos.

En el presente trabajo mostraremos cómo, a partir del análisis textual de una narración, se pueden detectar, con precisión suficiente, dificultades que pueden surgir en distintos ámbitos de lo que denominamos "mecanismos de coherencia". Esto nos permite crear una batería de preguntas control que detectan problemas de comprensión derivados de la incompleta o deficiente realización por parte del alumno, de los mecanismos de coherencia. En otro orden de actividad, las preguntas control pueden servir al mismo alumno para orientar su estrategia lectora.

A medio plazo, estas preguntas nos indican qué aspectos de la capacidad lectora del alumno deben ser trabajados. El lector puede no haber entendido un hecho concreto de la narración (por ejemplo, en el texto que 
presentamos a continuación, que el protagonista usa dos hojas de papel distintas) y sin embargo haber elaborado una historia perfectamente coherente. Esto quiere decir que hay que trabajar este aspecto del dominio de la definización y la anáfora.

Pretendemos, en cualquier caso, que lo que aquí practicamos con un texto narrativo, es aplicable a todos los que son vehículo del acto docente: el enunciado de un problema, la descripción de un fenómeno físico, las noticias del periódico... Estos textos a menudo ofrecen dificultades que no se detectan pero que inciden en la actividad que con ellos se pretende ejercer.

No trabajaremos, por obvias razones de espacio, el control del proceso de escritura.

El análisis citado irá precedido de la presentación de un marco teórico, donde se fija la terminología y se dan unas definiciones concretas. Este tipo de exposiciones teóricas, innecesario en otras disertaciones científicas, es inevitable en un ámbito científico muy joven todavía, donde las distintas escuelas no han impuesto aún una taxonomía única ni una metodología indiscutida.

A menudo, el enseñante se dará cuenta de que se ofrecen palabras nuevas para denominar sus intuiciones o, lo que le resultará más irritante, creerá asistir a un mero baile de neologismos. Es evidente que la lingüística del texto no parte de la nada y que, a menudo, las ciencias nuevas ponen gran énfasis en la terminología, pero creemos que resultará evidente que hay soluciones a muchos de sus antiguos problemas.

\section{Marco teórico: definiciones previas}

Ante todo, definiremos texto como una unidad comunicativa, tanto en la comunicación oral como en la escrita, en las lenguas naturales. Es difícil establecer una definición única y ajustada de lo que se entiende por coherencia, puesto que es un término muy amplio, puede considerarse desde muchos puntos de vista y no debe entenderse de un modo absoluto. Simplificando, cuando hablamos de coherencia textual nos referimos, por un lado, a las relaciones de cohesión que se establecen entre las unidades que constituyen un texto: relaciones cotextuales, entendiendo el cotexto como el conjunto lingüístico que rodea un elemento dado dentro de un texto. Estas relaciones, como ya veremos más adelante, no sólo son las que tradicionalmente considerábamos como de tipo sintáctico. Por otro lado, nos referimos también a las relaciones entre el texto y su situación de ocurrencia: relaciones contextuales, entendiendo que el contexto está formado por todos aquellos elementos de la situación en que se produce el texto, que determinan sistemáticamente su estructura y su interpretación, o 
bien que son determinados por esos textos. Los elementos que nosotros consideramos de manera sistemática cuando hablamos de contexto son: el enunciador, el destinatario, la intención, el lugar, el tiempo y el lugar social.

Así pues, podemos decir que un texto es coherente cuando se establece una relación de cohesión entre las unidades que lo constituyen y una relación de adecuación entre el texto y el contexto, entendiendo que esta adecuación incluye también la adecuación a la intención comunicativa del hablante.

\section{Mecanismos de coherencia textual}

La coherencia textual abarca muchos aspectos que actúan en diversos niveles. Proponemos abstraer los que nos parecen más relevantes para el análisis de un texto, en los siguientes mecanismos, sin que ello suponga agotar todos los aspectos de la coherencia textual. Advertimos que el orden de su exposición no implica un sistema de uso:

1. Tipología del texto

2. Mecanismos de repetición

3. Mecanismos de conexión

4. Mecanismos de progresión.

5. Implícitos.

6. Polifonía enunciativa.

7. Registros, Variedades y otras opciones estilísticas.

1. Las clasificaciones tipológicas que hallamos a nuestra disposición en los estudios de lingüística textual recurren a criterios y poseen intenciones diversos. Nosotros distinguiremos cuatro tipos de texto: narrativo, expositivo, descriptivo y conversacional, y para decidir lo que diferencia unos de otros, tomaremos en cuenta una serie de aspectos: la intención comunicativa (que incluye el tipo de acto de habla que se realiza), el género de discurso, la relación con la situación comunicativa, el lugar social de ocurrencia, el destinatario, la estructura, el tipo de conectores, de progresión temática y la polifonía enunciativa.

Así, la narración se distingue sobre todo porque no tiene una relación inmediata con la situación comunicativa (puede referirse a una situación comunicativa anterior, contemporánea o posterior a la del momento de producción, puede ser real o imaginada, no exige la intervención del interlocutor...), porque tiene una estructura determinada (las narraciones suelen organizarse alrededor del esquema básico: marco / complicación / resolución); en estos textos suelen predominar los conectores de tipo temporal y lógico, puesto que es importante la ordenación temporal de los sucesos y las relaciones de causa y efecto que los conectan y son textos que evidencian especialmente su polifonía. 
2. En cuanto a los mecanismos de repetición incluimos todos los fenómenos de referencia (mecanismos mediante los cuales los hablantes indican, a través de expresiones lingüísticas, las entidades del mundo real) y correferencia (expresiones lingǘsticas que refieren cotextualmente). En concreto, los mecanismos de repetición que consideramos son los siguientes: deixis y deixis textual, anáfora, elipsis, definización y cohesión léxica.

Estos conceptos han sido estudiados ya con anterioridad al desarrollo de la lingüística textual, pero ésta ha contribuido a dar explicaciones más satisfactorias a su funcionamiento.

3. Del mismo modo consideraremos los conectores que ya tenía en cuenta la gramática oracional teniendo en cuenta los valores cotextuales y contextuales que puedan tener: conjunciones, adverbios, locuciones adverbiales o sintagmas preposicionales, adjectivos numerales, demarcadores, algunas clases y ocurrencias de interjecciones, nombres, adjetivos, verbos y sintagmas, y la puntuación.

La presencia y/o repetición de ciertos tipos de conectores es característica de los diversos tipos de texto, de modo que, hasta cierto punto, podemos otorgar a los conectores un valor como marcadores tipológicos. Los clasificamos en tres grandes tipos: temporales, lógicos y ordenadores del espacio.

4. Por mecanismos de progresión entendemos la dinámica de repetición y renovación de información: las categorias Tema y Rema.

5. En la clase de los implícitos incluimos varios fenómenos: las implicaciones; las presuposiciones, entendidas como proposiciones que se dejan inferir de las frases de un texto, aunque lo transformemos negativa $\mathrm{o}$ interrogativamente, y que pueden servir para encadenar la frase siguiente; las inferencias, que se realizan al tener en cuenta un principio de cooperación que Grice sistematiza en cuatro Máximas Conversacionales: máxima de cantidad, de calidad de pertinencia y de orden. Segun estas máximas, los hablantes cooperan de forma que realizan sus enunciados tal como requiere una situación determinada. Es decir, ponen orden en sus palabras; claridad, verdad o honradez en sus aseveraciones; no dicen las cosas a medias, y dicen todo lo que es necesario dando todos los datos que sean necesarios y no más de los necesarios. Y, solapándose con las máximas, los sobreentendidos: implícitos que derivan del contexto y que se producen cuando uno de los interlocutores se pregunta no sobre el sentido del enunciado sino sobre la enunciación del otro, sobre el hecho de que se haya dicho algo en un contexto determinado. Muchas inferencias derivan del Conocimiento del Mundo: conjunto de saberes, tanto universales 
como particulares, que posee el enunciador; el Marco es el resultado de organizar estos conocimientos en estructuras.

6. Por polifonía enunciativa nos referimos al hecho de que, en una enunciación, se produce una concurrencia de voces provenientes de distintos enunciadores.

Si bién desde la observación empírica la enunciación es atribuida a un sólo sujeto, el análisis nos permite distinguir varias categorias, si consideramos al hablante en relación con el enunciado. Distinguimos el sujeto hablante (autor), del locutor (que denominaremos narrador en el texto narrativo) como responsable de la enunciación, ser que únicamente existe en el texto. Distinguimos una tercera categoría, la del enunciador propiamente dicho, que correspondería a aquellos seres que, aunque a veces no tienen una manifestación explícita en la enunciación (pueden carecer de la atribución de palabras precisas) sí que aparecen en la enunciación a través de proposiciones, opiniones... que no son atribuibles al locutor.

Existe todavía otra categoría: el conjunto de elecciones de temas y de técnicas narrativas, de valores y de modos de ver el mundo que se desprenden de la narración, que agrupamos bajo la denominación de autor implícito.

7. En este punto consideramos las diversas formas de utilización de la lengua, tanto en el aspecto convencional (registros lingüísticos) como en el individual (dialectos e idiolectos).

\section{Análisis de un texto literario:San Salvador de Peter Bichsel}

El siguiente no es un análisis exhaustivo, sino una muestra de cómo puede realizarse; recogemos por lo tanto algunos casos relevantes para cada tipo de mecanismo.

\section{TIPOLOGIA}

El texto que analizamos a continuación es un texto literario de tipo narrativo, que se puede clasificar dentro del género del cuento corto.

Respecto del emisor hay que tener en cuenta el papel relevante de la polifonía enunciativa en el texto narrativo. Así, el autor, como ser del mundo, no es propiamente responsable de la enunciación, sinó que és ta es atribuida a un narrador suscitado por el autor, el cual, a su vez, suscita otras voces, las de los enunciadores (los personajes hablando en discurso directo o "hablando" en indirecto libre, por ejemplo). De este modo, la relación entre el enunciado que constituye la narración y el autor es similar a la que se establece entre una cita y su citador: las palabras de la narración son enunciadas por el narrador y el papel del autor se limita a citar lo que el narrador ha dicho. En el caso de un texto literario como el que nos ocupa 
no hay que olvidar, además, que tanto el narrador como la narración son ficciones y, por lo tanto, a quien se cita y lo que se cita son un narrador y un texto puramente imaginados, que no han tenido nunca ninguna ocurrencia.

El carácter literario del texto provoca que las otras categorias contextuales también queden trastocadas. Hay que distinguir, pues, entre el contexto en el cual el autor ha citado la narración, el contexto -ficticio- en el que el narrador produce la narración, y, finalmente, el contexto en el que se sitúan los personajes, al cual remiten los deícticos y que puede incluir un destinatario ficticio. El contexto del lector en el momento de leer el texto diferirá también del contexto del autor y del narrador.

El lugar social de ocurrencia de este texto corresponde al de la institución literaria.

El destinatario es universal para cualquier texto literario. En cuanto al lector implícito, aquel creado por las estrategias del texto, se puede decir que se trata de un adulto, europeo, contemporáneo.

Por lo que se refiere a la intención, debemos referirnos de nuevo al carácter literario de este texto. Una enunciación literaria presenta una dualidad: en un sentido, el lenguaje es propuesto a la contemplación como mera estructura lingüística (intención estética), pero en otro sentido, el uso literario del lenguaje tiene también una intención comunicativa indirecta. Las intenciones estéticas y comunicativas concretas forman parte de lo que hemos llamado autor implícito.

El texto que nos ocupa, junto a una intención estética que debemos considerar de primer plano, tiene una intención argumentativa de tipo crítico hacia una determinada concepción de las relaciones sociales y familiares, hacia las relaciones del individuo con las instituciones y los mitos de una sociedad occidental desarrollada. Y si percibimos la ironía del autor implícito en la plasmación de conductas de unos personajes concebidos a imagen de nuestros conciudadanos, podemos predicar una intención crítica respecto de la alienación y los deseos frustrados de los individuos en estas sociedades.

La estructura de este texto sería la siguiente:

1. Marco (linea 1): alguien, en un tiempo pasado pero no muy lejano (el pluscuamperfecto indica que se trata de un tiempo anterior al de la narración pero no hay indicadores que precisen cual), realiza un acción que tiene por objecto la posesión de un objecto-símbolo: la pluma.

2. Complicación (2-34): Paul espera Hildegard mientras piensa, imagina y fantasea sobre acciones hipotéticas deseadas, por un lado, y sobre un futuro real inminente, por otro. 
El marco y la complicación explican, ordenados cronológicamente, los sucesos que ocurren antes de la llegada de Hildegard. Se dan en dos niveles: uno, donde se habla de los hechos y acciones que suceden en el exterior del personaje (el personaje prueba la pluma nueva -escribe y dibuja-, mira los periódicos, etc.), y otro que explica lo que sucede en el mundo interior del personaje (15-29) (piensa en lo que debe estar haciendo Hildegard y en lo que haría si al volver a casa descubriese que el se había ido).

3. Resolución (35-37): Hildegard vuelve a casa: en principio se corrobora la previsión de futuro real y se descartan casi todas las acciones hipotéticas. Se produce, sin embargo, un hecho mínimo inquietante: Hildegard "se aparta el pelo de la cara", realiza algo que antes sólo había previsto Paul en el cotexto de las acciones hipotéticas. Por metonimia es posible imaginar que el resto de acciones de Hildegard pensadas por Paul se realizan también. Se añade a esto que el texto "aquí hace demasiado frio..." no ha sido destruido y està todavía encima de la mesa, donde Hildegard podría verlo.

No hay ni coda ni una parte específicamente destinada a evaluación.

\section{POLIFONIA ENUNCIATIVA}

$\mathrm{El}$ aspecto polifónico es el que en esta narración tiene más relevancia, ya que toca directamente al juego de significaciones que el texto propone.

Para empezar, señalamos el aspecto básico de la polifonía de los textos narrativos: la distinción entre autor y narrador y la distinción entre el narrador y los enunciadores suscitados por éste ("Paul", "Hildegard"). El narrador y Paul son responsables de enunciaciones escritas; Paul de los textos que aparecen desde la linea 2 hasta la 9; el de 5-6 y el de 9, citado en discurso directo por el narrador. Hildegard es la enunciadora del texto oral de la linea 35, citado en discurso directo por el narrador.

Esta narración incluye, cita, siete textos que pasamos a enumerar.

El primer texto (1-37) es el del relato entero, enunciado por el narrador y focalizado desde una posición externa a los hechos, aunque accede al mundo interior del personaje. El destinatario puede coincidir con el lector implícito. No se puede hablar de un único acto de habla sino de un conjunto que se puede considerar integrado en un macroacto de habla, que podría ser el de relatar.

El enunciador del segundo texto (2-4) es también el narrador, que describe el texto que Paul está describiendo.

La existencia de este texto es dudosa porque no parece tener ninguna intención comunicativa ni parece ir dirigido a ningun destinatario. De todos modos, las pruebas que realiza Paul con la pluma simulan lo que serían varios enunciados: dos direcciones, unas iniciales y unas lineas onduladas. La estructura de este hipotético texto reproduce básicamente lo que sería una 
carta: dirección (de los padres), remitente (dirección de Paul), contenido (lineas onduladas) y firma (iniciales), aunque no sigue las disposiciones espaciales propias de una carta.

El contenido, que constaría de un enunciado icónico -las lineas onduladas-, sólo puede tener significado si establecemos una relación de coherencia cotextual con el próximo texto.

El tercer texto (5-6 y 9) es un texto escrito, asumido, como cita en discurso directo, dentro del texto del narrador, que tiene por enunciador a Paul. El destinatario será, más tarde, Hildegard y el texto es una argumentación donde Paul que se va y la razón de ello. De momento se trata de un texto expositivo, que más tarde se manifestará como mensaje.

El cuarto texto (31-32), las instrucciones de uso de la pluma, citadas en discurso directo, se presenta sin entrecomillado, pero sin conservar los caracteres tipográficos originales. Podría tratarse de un fragmento del discurso mental, interno, del propio protagonista, que reproduciría literalmente las frases del texto de las instrucciones que, a la sazón, estaría leyendo.

El quinto texto (35-36) es oral y se asume como cita en discurso directo dentro del texto del narrador. La enunciadora es Hildegard y el destinatario Paul.

El sexto texto (15-29), si es que podemos considerar que se trata de un texto, correspondería al monólogo interior y al conjunto de los pensamientos del protagonista, asumido en discurso indirecto libre por el narrador. El enunciador sería Paul y también sería Paul el destinatario, como en todo monólogo.

La existencia del séptimo texto (24-25) ("alguna cosa habría pasado") es aun más dudosa que la del anterior porque corresponde a un fragmento del discurso indirecto libre que reproduce el monólogo interno del protagonista, Paul, el cual, a su vez, reproduciría el monólogo interior de Hildegard, también en discurso indirecto libre. Paul prevé lo que pensará Hildegard cuando vuelva y haya leído el texto "aquí hace demasiado frío...". Así pues, tanto la situación como las palabras son puras hipótesis de Paul.

En definitiva, lo que nos lleva a considerar la existencia de estos dos últimos textos es la presencia del discurso indirecto libre, en tanto que es te permite reproducir proposiciones, percepciones e incluso expresiones internas de los personajes, aunque sean asumidas por el narrador.

\section{MECANISMOS DE REPETICION}

Nos referiremos únicamente a algunos aspectos de la deixis, de la cohesión léxica y de la definización que nos parecen destacables en este texto. 
Deixis. Recordemos que, tal como decíamos al principio al hablar de las características enunciativas del texto literario, los deícticos estan anclados en el contexto ficticio, creado.

Nos referimos solamente a los deícticos que aparecen en lo que hemos considerado $6^{\circ}$ texto, enunciado en discurso indirecto libre. Tal como es característico en este modo de discurso, los deícticos pertenecen en parte al narrador (tiempos verbales, $3^{\circ}$ persona), y en parte al personaje (expresiones referentes al aquí y ahora de la enunciación). Estas últimas presentan algunas particularidades. De los tres "ahora" (17, 18 y 21) que leemos, dos aparecen ligados bien a acciones puntuales, que introducen un cambio en el estado de cosas (apagar la radio, 17), o bien a percepciones irrepetibles (que son las nueve, 21). El "ahora" de 18 no se relaciona con un hecho ni con un cambio en el estado de cosas, ni, en principio, se marca la diferencia con ningun estado de las cosas "antes" (ni "después"): la hoja doblada ya estaba encima de la mesa desde que apareció en la linea 4, y no ha cambiado de lugar o, por lo menos, el cotexto no permite entender otra cosa

Existe una posibilidad de que la expresión "estaba ahora" (18) permita inferir un cambio en el marco respecto de un "antes" y un "después", compatible con el cotexto: el que se da cuando lo consideramos ligado al movimiento de la conciencia del personaje Paul. Veamos la situación: Paul, depués de escribir el texto 5-6 y 9 se distrae en diversas ocupaciones y pensamientos (10-17) hasta que redescubre su escrito anterior (18-19): el "ahora" de 18 marca este redescubrimiento por parte de la conciencia de Paul. Se trata de un momento importante porque en ese momento Paul se convierte en lector de su propio escrito, otorga una intención e imagina un destinatario y, por lo tanto, el escrito de 5-6 y 9 se convierte propiamente en texto. La lectura que proponemos de este "ahora" es coherente con la función del discurso indirecto libre que señalábamos: el relato de la conciencia de los personajes.

Más adelante, al hablar de implícitos, analizaremos otro deíctico relevante: "aquí hace demasiado..." (7-8).

Cohesión léxica. En este texto, la cohesión pone de manifiesto la existencia de algunos marcos. Así, "viaje" es un marco (tópico) no explicitado que incluye desde "camisas" (23) hasta "América del Sur" (6). A su vez, "América del Sur" incluye desde el título (San Salvador, que no se encuentra en América del Sur) o "lineas onduladas" (3), o "demasiado frío" (5) -por antítesis-, hasta "palmeras" (33). La capacidad que tienen estos marcos para aglutinar expresiones aparentemente alejadas es un elemento fundamental de esta narración. Un caso parecido, quizás menos inesperado, 
és el de "trazar" (2) y sus complementos: "iniciales, dirección, lineas onduladas" (3-4), etc.

En realidad se dan tres grandes marcos implícitos: "viajar", "escribir" y "hogar familiar" (este último da coherencia desde el marco "mesa" hasta "los niños" (36) y permite algunas inferencias importantes, como ya veremos). El marco viaje y el marco escribir se interseccionan en "lineas onduladas" y, desde luego, en "me voy a América del Sur". La recurrencia de los marcos es muy importante de cara al juego de implícitos, $\mathrm{y}$, en este texto, por cuanto destaca el carácter tópico y por lo tanto previsible del universo recreado por la narración.

Definización. Es también un elemento importante de coherencia en este texto. Señalamos, por ejemplo, "una pluma estilográfica" (1), "u na hoja" (2) y "una hoja nueva" (4): a "una hoja" le corresponde "la hoja de líneas onduladas" (12); a "una nueva hoja" le corresponde "la hoja" (7), "la hoja doblada" (18), "el papel" (33).

Gran parte de los substantivos aparecen por primera vez ya acompañados del artículo, es decir, como elementos ya conocidos, gracias a la recurrencia de los marcos que acabamos de mencionar. Respecto a esto, hay una definización un tanto complicada "El Löewen" (26), que comentaremos al hablar de los implícitos.

Mecanismos de conexión. La presencia de conectores de tipo temporal es abundante, tal como es previsible en un texto narrativo. Esta abundancia se convierte aquí en una característica estilística. Observemos que los conectores temporales son básicamente dos: "después" y "ahora". El primero, que corresponde al mundo narrado desde el punto de vista del narrador, conecta las oraciones y refuerza el carácter de linealidad temporal de la serie de actos que realiza Paul. El segundo aparece en el fragmento en discurso indirecto libre y marca, también, la sucesión lineal de presentes en la conciencia del personaje.

\section{IMPLiCITOS}

Nos detenemos sólo en algunos implícitos importantes para el sentido global del texto y por tanto para su coherencia.

De entrada podemos dar cuenta de diversas inferencias praxeológicas sobre el marco, o mejor los marcos del contexto evocado y, a partir de ellos $\mathrm{y}$ de lo que se nos dice que hace el personaje, podemos inferir cosas como falta de proyectos concretos, aburrimiento, etc.; o, teniendo en cuenta también la definización en los niños (35-36), que se trata de pareja con hijos.

En la línea 5, la expresión "aquí hace demasiado frío para mi" presupone "aquí hace frío". Si pensamos que la alternativa geográfica es América del Sur, "aquí" debe abrazar evidentemente un espacio más amplio que el de la 
habitación donde sucede la enunciación: el país o Europa Central. Y si observamos la ocurrencia -hipotética, en la mente de Paul- del texto (20-22), "aquí" designa también un lugar y todo un marco social/familiar. Esta deixis sirve para explicar el mecanismo por el cual se produce el sobreentendido más importante de "San Salvador": al preguntarnos porqué Paul dice lo que dice en el contexto determinado que se nos presenta (es decir, en el cotexto de la narración incluídas las inferencias que nuestro conocimiento del mundo nos permite realizar), inferimos que "frío" no denota una sensación física sino más bien la imagen de un malestar psicológico, El mensaje completo implicita un deseo de huída hacia un mundo alternativo y expresa ilocutoriamente un aviso o una amenaza.

Otro implícito destacable es el que se produce como hipótesis alternativa del referente de "Löwen" (25-26). Aquí el conocimiento del mundo juega un papel importante. De entrada, si sabemos que el Löwen cierra un dia determinado de la semana, según la máxima de cantidad inferimos que el resto de los días no está cerrado. Nuestro conocimiento del mundo nos dice que lo más probable es que se trate de un local público -que probablemente abre todos los días excepto uno; que es un lugar frecuentado por las noches por el personaje, como alternativa a quedarse en casa y, por lo tanto, que se trata de un lugar de recreo, un pub o algo similar.

Otra inferencia que se puede extraer y que es importante para la interpretación del texto, es que probablemente, si el Löwen estuviera abierto, Paul no estaría en casa sin saber muy bién qué hacer y los hechos narrados no se habrían producido.

\section{Preguntas de control}

Las preguntas que a continuación proponemos merecen algún comentario. En primer lugar, y en el sentido que hemos expuesto a lo largo de este artículo, lo que sigue no quiere ser un control de la comprensión lectora del alumno. En segundo lugar, el error en la elección de respuesta no descalifica, siquiera parcialmente, la competencia textual del alumno: una determinada opción, aunque no sea la óptima bien puede constituir en sí misma un elemento de un lectura coherente, distinta en algún aspecto de la nuestra, y perfectamente admisible por el texto -la pregunta 7 puede ser un ejemplo de ello. El profesor extraerá las consecuencias pertinentes

En algunos casos resulta muy difícil discriminar el control de un mecanismo de coherencia concreto, como queda constancia en las preguntas 10, 11 y 12 .

Finalmente debemos señalar que el cuestionario que sigue sólo pretende ser una muestra, una sugerencia de procedimiento que puede ser ampliado y 
perfeccionado desde nuestro propio análisis y, obviamente, una vez sometido a experimentación.

\section{De la estructura}

1/ Al llegar a casa, Hildegard...

a/ No puede leer el escrito de Paul porque Paul lo ha roto b/ No lee el escrito de Paul. c/ No sabemos si lee o no el escrito de Paul.

Como señalábamos en el análisis, es importante destacar, a través de la respuesta $\mathrm{c} /$, la pequeña incertidumbre de un final no totalmente cerrado

\section{Sobre polifonía y actos de habla}

2/ (Sobre la intención comunicativa) Paul escribe su propia dirección y la de sus padres.

a/ Quiere escribir una carta a sus padres. b/ Quiere probar la pluma estilográfica. c/ Quiere escribir una carta a Hildegard.

Con la respuesta $\mathrm{b} /$ comprobamos que en el momento del cuento que indica el enunciado de la pregunta no existe intención comunicativa -si no es de manera totalmente inconsciente- por parte de Paul y que, por lo tanto, no podemos considerar el escrito como la realización de un acto ilocutivo.

3/ (Sobre actos de habla) Cuando Paul escribe "Aquí hace demasiado calor...América del Sur" (5 y 6)

a/ Tan sólo está probando la pluma. b/ Está avisando a sus padres de su próxima partida a América del Sur. c/ Está decidido a irse y avisa a Hildegard de que se va.

Aunque podría caber c/ debemos descartarla puesto que Hildegard no aparece en la mente de Paul hasta más adelante. En rigor sólo podemos asegurar a/ ya que el acto de la firma se dilata un tanto en el tiempo (9)

4/ (Sobre polifonía y el estilo indirecto libre) ¿Qué indica "a pesar de todo... camisas" (23)?

a/ Que Paul piensa que Hildegard contaría las camisas. b/ Que el narrador asegura que cuando llegue Hildegard contará las camisas. c/

Que Hildegard llega y cuenta las camisas.

5/ (Sobre polifonía -y también estilo indirecto libre) ¿Quién se exclama "Alguna cosa habría pasado"? (23-24)

a/ Paul. b/ Hildegard. c/ Quien narra la historia.

Notemos que la exclamación pertenece al conjunto de reacciones de Hildegard previstas por Paul: es Hildegard, como personaje dentro del "texto" del monólogo de Paul, la responsable de tal reacción expresiva.

\section{De los implícitos}

6/ (De sobreentendidos) ¿Cuál de las siguientes afirmaciones es la correcta? 
a/ Hildegard telefoneará al Löwen. b/ Es miércoles. c/ Paul frecuenta el Löwen los miércoles.

De hecho la única inferencia posible es b/. La elección de a/, aparentemente posible, debe descartarse por cuanto, en primer lugar, c/ es mucho más segura y, en segundo lugar, a/ está formulada en indicativo y no en condicional.

7/ (De sobreentendidos) ¿Cuál de las siguientes afirmaciones refleja mejor lo que nos dice el cuento?:

a/ Paul desea irse porque pasa frío en su casa. b/ Paul desea irse porque vive en un país frío. c/ Paul desea irse porque hay algo en su vida que le desagrada.

Las diversas respuestas representan diferentes lecturas. En cualquier caso, si no se da c/ cabe la posibilidad que encontremos dificultades al pasar a un nivel interpretativo superior.

8/ (Sobre el marco) ¿Qué palabra va a continuación de la siguiente secuencia: "Papelería", "capuchón", "líneas onduladas"...?

a/ "Instrucciones de uso". b/ "Löwen". c/ Ninguna, son palabras inconexas.

\section{Sobre definización}

9/ La hoja de líneas onduladas de 13-14 es también:

a/ "una hoja nueva" de la línea 4. b/ "una hoja" de la línea 2. c/ "la hoja" de la línea 19.

\section{Sobre deixis}

10/ (Deícticos e implícitos) En 18 se dice que "...encima de la mesa, estaba ahora la hoja doblada" porque...

a/ Antes no estaba encima de la mesa. b/ Paul la ha recuperado del cenicero. c/ Paul se había olvidado de su presencia.

\section{Sobre la elipsis}

11/ (Elipsis e implícitos) ¿Cuál es la información adecuada?

a/ Paul y Hildegard son hermanos. b/ Paul y Hildegard son marido y mujer. c/ No podemos hacernos una idea del parentesco que tienen.

Cabe descartar c/ por los modalizadores que presenta su enunciado: justamente es hacia b/ que nos lleva el texto. La alternativa, en este caso, implica una gran variación del sentido del cuento, sea éste el que fuere.

12/ (Elipsis e implícitos) Elige la información más apropiada:

a/ El Löwen es un local público donde Paul pasa algunos ratos. b/ El Löwen es el lugar de trabajo de Paul. c/ Ni a/, ni b/.

Aquí la inferencia es compleja -véase el análisis- por lo que insistimos en que se trata de elegir la mejor. 


\section{San Salvador}

Se había comprado un pluma estilográfica.

Después de haber trazado varias veces su firma sobre una hoja, después de haber escrito sus iniciales, su dirección, unas líneas onduladas, la dirección de sus padres, cogió una hoja nueva, la dobló con cuidado y escribió: "aquí hace demasiado frío para mi" -y después- "me voy a América del Sur". Después se detuvo, colocó el capuchón sobre la pluma, miró la hoja y observó cómo la tinta se secaba y se oscurecía (en la papelería le habían garantizado que se volvía negra), después volvió a coger la pluma y añadió su nombre: "Paul".

Después permaneció sentado.

Más tarde levantó los periódicos de la mesa, y al hacerlo, dio un vistazo a la cartelera y pensó en cualquier cosa, apartó el cenicero, rompió la hoja de líneas onduladas, vació la pluma y la volvió a llenar. Para la sesión de cine ya era demasiado tarde.

El ensayo del coro de la Iglesia duraba hasta las nueve, a las nueve y media Hildegard ya habría vuelto. Esperaba a Hildegard. Mientras, música de la radio. Ahora apagó la radio.

Encima de la mesa, en medio de la mesa, estaba ahora la hoja doblada, y sobre la hoja, en escritura negroazulada, su nombre, Paul.

También ponía "aquí hace demasiado frío para mi".

Hildegard llegaría, pues, a las nueve y media. Ahora eran las nueve. Leería su aviso, se asustaría, probablemente no se creería aquello de América del Sur, a pesar de todo contaría las camisas en el cajón: alguna cosa habría pasado.

Telefonearía al "Löwen".

El Löwen está cerrado los miércoles.

Sonreiría, se desesperaría y se resignaría, quizás.

Se apartaría el cabello de la cara, reseguiría con el dedo anular de la mano izquierda las sienes, entonces se desabrocharía lentamente el abrigo.

Después permaneció sentado, pensó a quién le podía enviar una carta, leyó de nuevo las instrucciones de uso para la estilográfica (girar un poco a la derecha), también leyó el texto en francés, comparó el inglés con el alemán, volvió a ver el papel, pensó en palmeras, pensó en Hildegard.

Permanecía sentado.

Y a las nueve y media llegó Hildegard y preguntó: "¿Duermen los niños?".

Se apartó el cabello de la cara.

\section{Bibliografía:}

BERNARDEZ, E. (1982). Introducción a la lingüistica del texto. Ed. EspasaCalpe, Madrid. 
BRONCKART, J. P.,i altres (1986). Le fonctionnenment des discours. Delachaux et Niestlé, París

CERVONI, J. (1987). L'énonciation. PUF, París.

DUCROT, O. (1972). Dire et ne pas dire. París.

KERBRAT-ORECCHIONI, C. (1986). L'implicite. Armand Colin, París.

LEVINSON,S. C. (1983). Pragmática. Teide, Barcelona, 1990

REYES, G. (1984). Polifonía textual. La citación en el relato literario. Gredos, Madrid

VAN DIJK, T. A. (1978). La ciencia del texto. Ed. Paidós, Barcelona, 1983. 